\title{
Inequalities for the composition of Green's operator and the potential operator
}

\author{
Zhimin Dai ${ }^{1 *}$, Yuming Xing ${ }^{1}$, Shusen Ding ${ }^{2}$ and Yong Wang ${ }^{1}$
}

"Correspondence: zmdai@yahoo.cn 'Department of Mathematics, Harbin Institute of Technology, Harbin, China

Full list of author information is available at the end of the article

\begin{abstract}
We first establish the $L^{p}$-norm inequalities for the composition of Green's operator and the potential operator. Then we develop the $L^{\varphi}$-norm inequalities for the composition in the $L^{\varphi}$-averaging domains. Finally, we display some examples for applications.

MSC: Primary 35J60; secondary 31B05; 58A10; 46E35

Keywords: differential forms; $L^{p}$-norm; the Luxemburg norm; Green's operator; the potential operator
\end{abstract}

\section{Introduction}

The purpose of this paper is to derive some inequalities for the composition of Green's operator $G$ and the potential operator $P$ applied to differential forms. The differential forms are extensions of functions and can be used to describe various systems of PDEs, physics, theory of elasticity, quasiconformal analysis, etc. In the meanwhile, Green's operator and the potential operator are of considerable importance in the study of potential theory and nonlinear elasticity; see [1-6] for more properties of these two operators. In many situations, the process to study solutions of PDEs involves estimating the various norms of the operators. Bi defined a potential operator $P$ applied to differential forms in [1]. However, the study on the composition of the potential operator and other operators has just begun. Hence, we are motivated to establish some inequalities for the composite operator $G \circ P$ applied to differential forms.

Now we introduce some notations. Unless otherwise indicated, we always use $\Theta$ to denote an open subset of $\mathbb{R}^{n}(n \geq 2)$, and let $O$ be a ball in $\mathbb{R}^{n}$. Let $\rho O$ denote the ball with the same center as $O$ and $\operatorname{diam}(\rho O)=\rho \operatorname{diam}(O), \rho>0$. A weight $w(x)$ is a nonnegative locally integrable function in $\mathbb{R}^{n} .|D|$ is used to denote the Lebesgue measure of a set $D \subset \mathbb{R}^{n}$. Let $\wedge^{\ell}=\wedge^{\ell}\left(\mathbb{R}^{n}\right), \ell=0,1, \ldots, n$, be the linear space of all $\ell$-forms $\hbar(x)=\sum_{J} \hbar_{J}(x) d x_{J}=\sum_{J} \hbar_{j_{1} j_{2} \cdots j_{\ell}}(x) d x_{j_{1}} \wedge d x_{j_{2}} \wedge \cdots \wedge d x_{j_{\ell}}$ in $\mathbb{R}^{n}$, where $J=\left(j_{1}, j_{2}, \ldots, j_{\ell}\right)$, $1 \leq j_{1}<j_{2}<\cdots<j_{\ell} \leq n$, are the ordered $\ell$-tuples. Moreover, if each of the coefficients $\hbar_{J}(x)$ of $\hbar(x)$ is differential on $\Theta$, then we call $\hbar(x)$ a differential $\ell$-form on $\Theta$ and use $D^{\prime}\left(\Theta, \wedge^{\ell}\right)$ to denote the space of all differential $\ell$-forms on $\Theta . C^{\infty}\left(\Theta, \wedge^{\ell}\right)$ denotes the space of smooth $\ell$-forms on $\Theta$. The exterior derivative $d: D^{\prime}\left(\Theta, \wedge^{\ell}\right) \rightarrow D^{\prime}\left(\Theta, \wedge^{\ell+1}\right), \ell=0,1, \ldots, n-1$, is given by

$$
d \hbar(x)=\sum_{i=1}^{n} \sum_{J} \frac{\partial \hbar_{j_{1} j_{2} \cdots j_{\ell}}(x)}{\partial x_{i}} d x_{i} \wedge d x_{j_{1}} \wedge d x_{j_{2}} \wedge \cdots \wedge d x_{j_{\ell}}
$$


for all $\hbar \in D^{\prime}\left(\Theta, \Lambda^{\ell}\right)$ and the Hodge codifferential operator $d^{\star}$ is defined as $d^{\star}=(-1)^{n \ell+1} \star$ $d \star: D^{\prime}\left(\Theta, \Lambda^{\ell+1}\right) \rightarrow D^{\prime}\left(\Theta, \Lambda^{\ell}\right)$, where $\star$ is the Hodge star operator. $L^{p}\left(\Theta, \wedge^{\ell}\right)$ is a Banach space with the norm

$$
\|\hbar\|_{p, \Theta}=\left(\int_{\Theta}|\hbar(x)|^{p} d x\right)^{1 / p}=\left(\int_{\Theta}\left(\sum_{J}\left|\hbar_{J}(x)\right|^{2}\right)^{p / 2} d x\right)^{1 / p}<\infty .
$$

For a weight $w(x)$, we write $\|\hbar\|_{p, \Theta, w}=\left(\int_{\Theta}|\hbar|^{p} w(x) d x\right)^{1 / p}$. From [7], if $\hbar$ is a differential form in a bounded convex domain $\Theta$, then there is a decomposition

$$
\hbar=d(T \hbar)+T(d \hbar)
$$

where $T$ is called a homotopy operator. Furthermore, we can define the $\ell$-form $\hbar_{\Theta} \in$ $D^{\prime}\left(\Theta, \wedge^{\ell}\right)$ by

$$
\hbar_{\Theta}= \begin{cases}|\Theta|^{-1} \int_{\Theta} \hbar(y) d y, & \ell=0 \\ d(T \hbar), & \ell=1, \ldots, n .\end{cases}
$$

for all $\hbar \in L^{p}\left(\Theta, \wedge^{\ell}\right), 1 \leq p<\infty$.

With respect to the nonhomogeneous $A$-harmonic equation for differential forms, we indicate the general form as follows:

$$
d^{\star} A(x, d \hbar)=B(x, d \hbar)
$$

where $A: \Theta \times \wedge^{\ell}\left(\mathbb{R}^{n}\right) \rightarrow \wedge^{\ell}\left(\mathbb{R}^{n}\right)$ and $B: \Theta \times \wedge^{\ell}\left(\mathbb{R}^{n}\right) \rightarrow \wedge^{\ell-1}\left(\mathbb{R}^{n}\right)$ satisfy the conditions: $|A(x, \eta)| \leq a|\eta|^{p-1}, A(x, \eta) \cdot \eta \geq|\eta|^{p}$ and $|B(x, \eta)| \leq b|\eta|^{p-1}$ for almost every $x \in \Theta$ and all $\eta \in \wedge^{\ell}\left(\mathbb{R}^{n}\right)$. Here $a, b>0$ are some constants and $1<p<\infty$ is a fixed exponent associated with (1.5). A solution to (1.5) is an element of the Sobolev space $W_{\operatorname{loc}}^{1, p}\left(\Theta, \wedge^{\ell-1}\right)$ such that

$$
\int_{\Theta} A(x, d \hbar) \cdot d \psi+B(x, d \hbar) \cdot \psi=0
$$

for all $\psi \in W_{\text {loc }}^{1, p}\left(\Theta, \wedge^{\ell-1}\right)$ with compact support, where $W_{\text {loc }}^{1, p}\left(\Theta, \wedge^{\ell-1}\right)$ is the space of $\ell$-forms whose coefficients are in the Sobolev space $W_{\text {loc }}^{1, p}(\Theta)$.

Recently, Bi extended the definition of the potential operator to the set of all differential forms in [1]. For any differential $\ell$-form $\hbar(x)=\sum_{J} \hbar_{J}(x) d x_{J}$, the potential operator $P$ is defined by

$$
P \hbar(x)=P\left(\sum_{J} \hbar_{J}(x) d x_{J}\right)=\sum_{J} P\left(\hbar_{J}(x)\right) d x_{J}=\sum_{J} \int_{\Theta} K(x, y) \hbar_{J}(y) d y d x_{J},
$$

where the kernel $K(x, y)$ is a nonnegative measurable function defined for $x \neq y, \hbar_{J}(x)$ is defined on $\Theta \subset \mathbb{R}^{n}$ and the summation is over all ordered $\ell$-tuples $J$. For more results related to the potential operator $P$, see $[1,8,9]$. 


\section{The $L^{p}$-norm inequalities}

In this section, we establish the $L^{p}$-norm inequality for the composite operator $G \circ P$ and obtain the $A_{r, \lambda}(\Theta)$-weight version of the inequality. We need the following definitions and lemmas.

In [5], Ding gives the following $L^{p}$-norm inequality for Green's operator.

Lemma 2.1 Let $\hbar \in L^{p}\left(\Theta, \wedge^{\ell}\right)$ be a smooth form in a bounded domain $\Theta, \ell=1, \ldots, n, 1<$ $p<\infty$ and $G$ be Green's operator. Then there exists a constant $C$, independent of $\hbar$, such that

$$
\left\|G(\hbar)-(G(\hbar))_{O}\right\|_{p, O} \leq C|O| \operatorname{diam}(O)\|\hbar\|_{p, O}
$$

for all balls $O \subset \Theta$.

Definition 2.2 ([3]) A pair of weights $\left(w_{1}(x), w_{2}(x)\right)$ satisfies the $A_{r, \lambda}(\Theta)$-condition in a set $\Theta \subset \mathbb{R}^{n}$, write $\left(w_{1}(x), w_{2}(x)\right) \in A_{r, \lambda}(\Theta)$ for some $\lambda \geq 1$ and $1<r<\infty$ with $\frac{1}{r}+\frac{1}{r^{\prime}}=1$, if

$$
\sup _{O \subset \Theta}\left(\frac{1}{|O|} \int_{O} w_{1}^{\lambda} d x\right)^{\frac{1}{\lambda r}}\left(\frac{1}{|O|} \int_{O}\left(\frac{1}{w_{2}}\right)^{\frac{\lambda r^{\prime}}{r}} d x\right)^{\frac{1}{\lambda r^{\prime}}}<\infty .
$$

The following definition is introduced in [9].

Definition 2.3 A kernel $K$ on $\mathbb{R}^{n} \times \mathbb{R}^{n}(n \geq 2)$ satisfies the standard estimates if there exist $\alpha, 0<\alpha \leq 1$, and a constant $C$ such that, for all distinct points $x$ and $y$ in $\mathbb{R}^{n}$ and all $z$ with $|x-z|<\frac{1}{2}|x-y|$ :

(1) $|K(x, y)| \leq C|x-y|^{-n}$;

(2) $|K(x, y)-K(z, y)| \leq C\left|\frac{x-z}{x-y}\right|^{\alpha}|x-y|^{-n}$;

(3) $|K(z, y)-K(x, y)| \leq C\left|\frac{x-z}{x-y}\right|^{\alpha}|x-y|^{-n}$.

The following two-weight norm inequality for the potential operator $P$ applied to differential forms appears in [1].

Lemma 2.4 ([1]) Let $\hbar \in L^{p}\left(\Theta, \wedge^{\ell}\right), \ell=0,1, \ldots, n, 1<p<\infty$, be a differential form in a domain $\Theta$ and $P$ be the potential operator defined in (1.7) with the kernel $K(x, y)$ satisfying the condition (1) of the standard estimates (2.3). Assume that $\left(w_{1}, w_{2}\right) \in A_{r, \lambda}(\Theta)$ for some $\lambda \geq 1$ and $1<r<\infty$. Then there exists a constant $C$, independent of $\hbar$, such that

$$
\|P(\hbar)\|_{p, \Theta, w_{1}} \leq C\|\hbar\|_{p, \Theta, w_{2}} .
$$

Remark If let $w_{1}=w_{2}=1$ in (2.4), then (2.4) reduces to the following inequality:

$$
\|P(\hbar)\|_{p, \Theta} \leq C\|\hbar\|_{p, \Theta} .
$$


Theorem 2.5 Let $\hbar \in L^{p}\left(\Theta, \wedge^{\ell}\right), \ell=0,1, \ldots, n, 1<p<\infty$, be a differential form in a bounded convex domain $\Theta \subset \mathbb{R}^{n}, P$ be the potential operator defined in (1.7) with the kernel $K(x, y)$ satisfying the condition (1) of the standard estimates (2.3) and $G$ be Green's operator. Then there exists a constant $C$, independent of $\hbar$, such that

$$
\left\|G(P(\hbar))-(G(P(\hbar)))_{O}\right\|_{p, O} \leq C|O| \operatorname{diam}(O)\|\hbar\|_{p, O}
$$

for all balls $O$ with $O \subset \Theta$.

Proof By Lemma 2.1 and (2.5), we have

$$
\begin{aligned}
\left\|G(P(\hbar))-(G(P(\hbar)))_{O}\right\|_{p, O} & \leq C_{1}|O| \operatorname{diam}(O)\|P(\hbar)\|_{p, O} \\
& \leq C_{2}|O| \operatorname{diam}(O)\|\hbar\|_{p, O} .
\end{aligned}
$$

We complete the proof of Theorem 2.5.

Lemma 2.6 Let $0<\alpha, \beta<\infty$ and $1 / s=1 / \alpha+1 / \beta$. Iff and $g$ are two measurable functions on $\mathbb{R}^{n}$, then

$$
\|f g\|_{s, \Theta} \leq\|f\|_{\alpha, \Theta} \cdot\|g\|_{\beta, \Theta}
$$

for any $\Theta \subset \mathbb{R}^{n}$.

Lemma 2.7 ([10]) Let $\hbar$ be a solution of the nonhomogeneous A-harmonic equation (1.5) in a domain $\Theta$ and $0<s, t<\infty$. Then there exists a constant $C$, independent of $\hbar$, such that

$$
\|\hbar\|_{s, O} \leq C|O|^{(t-s) / s t}\|\hbar\|_{t, \rho O}
$$

for all balls $O$ with $\rho O \subset \Theta$, where $\rho>1$ is a constant.

Based on Theorem 2.5, we obtain the following $A_{r, \lambda}(\Theta)$-weight version of inequality (2.6).

Theorem 2.8 Let $\hbar \in L^{p}\left(\Theta, \wedge^{\ell}\right), \ell=0,1, \ldots, n, 1<p<\infty$, be a solution of the nonhomogeneous A-harmonic equation (1.5) in a bounded convex domain $\Theta, P$ be the potential operator defined in (1.7) with the kernel $K(x, y)$ satisfying the condition (1) of the standard estimates (2.3) and $G$ be Green's operator. Assume that $\left(w_{1}(x), w_{2}(x)\right) \in A_{r, \lambda}(\Theta)$ for some $\lambda \geq 1$ and $1<r<\infty$. Then there exists a constant $C$, independent of $\hbar$, such that

$$
\left\|G(P(\hbar))-(G(P(\hbar)))_{O}\right\|_{p, O, w_{1}^{\alpha}} \leq C|O| \operatorname{diam}(O)\|\hbar\|_{p, \rho O, w_{2}^{\alpha}}
$$

for all balls $O$ with $\rho O \subset \Theta$, where $\rho>1$ and $\alpha$ are two constants with $0 \leq \alpha<\lambda$. 
Proof Let $s=\lambda p /(\lambda-\alpha)$ and $t=\lambda p /(\lambda+\alpha(r-1))$, thus $0<t<p<s$. Using Theorem 2.5, Lemma 2.6 with $1 / p=1 / s+(s-p) /(s p)$ and Lemma 2.7 , we obtain

$$
\begin{aligned}
& \left\|G(P(\hbar))-(G(P(\hbar)))_{O}\right\|_{p, O, w_{1}^{\alpha}} \\
& =\left(\int_{O}\left|G(P(\hbar))-(G(P(\hbar)))_{O}\right|^{p} w_{1}^{\alpha} d x\right)^{1 / p} \\
& =\left(\int_{O}\left(\left|G(P(\hbar))-(G(P(\hbar)))_{O}\right| w_{1}^{\alpha / p}\right)^{p} d x\right)^{1 / p} \\
& \leq\left(\int_{O}\left|G(P(\hbar))-(G(P(\hbar)))_{O}\right|^{s} d x\right)^{1 / s}\left(\int_{O} w_{1}^{s \alpha /(s-p)} d x\right)^{(s-p) /(s p)} \\
& =\left\|G(P(\hbar))-(G(P(\hbar)))_{O}\right\|_{s, O}\left(\int_{O} w_{1}^{\lambda} d x\right)^{\alpha /(\lambda p)} \\
& \leq C_{1}|O| \operatorname{diam}(O)\|\hbar\|_{s, O}\left(\int_{O} w_{1}^{\lambda} d x\right)^{\alpha /(\lambda p)} \\
& \leq C_{2}|O| \operatorname{diam}(O)|O|^{(t-s) /(s t)}\|\hbar\|_{t, \rho O}\left(\int_{O} w_{1}^{\lambda} d x\right)^{\alpha /(\lambda p)} .
\end{aligned}
$$

Applying Lemma 2.6 with $1 / t=1 / p+(p-t) /(p t)$ yields

$$
\begin{aligned}
\|\hbar\|_{t, \rho O} & =\left(\int_{\rho O}|\hbar|^{t} d x\right)^{1 / t}=\left(\int_{\rho O}\left(|\hbar| w_{2}^{\alpha / p} w_{2}^{-\alpha / p}\right)^{t} d x\right)^{1 / t} \\
& \leq\left(\int_{\rho O}|\hbar|^{p} w_{2}^{\alpha} d x\right)^{1 / p}\left(\int_{\rho O}\left(\frac{1}{w_{2}}\right)^{\alpha t /(p-t)} d x\right)^{(p-t) /(p t)} \\
& =\|\hbar\|_{p, \rho O, w_{2}^{\alpha}}\left(\int_{\rho O}\left(\frac{1}{w_{2}}\right)^{\lambda /(r-1)} d x\right)^{\alpha(r-1) /(\lambda p)} .
\end{aligned}
$$

Note that $\left(w_{1}(x), w_{2}(x)\right) \in A_{r, \lambda}(\Theta)$, therefore

$$
\begin{aligned}
& \left(\int_{O} w_{1}^{\lambda} d x\right)^{\alpha /(\lambda p)}\left(\int_{\rho O}\left(\frac{1}{w_{2}}\right)^{\lambda /(r-1)} d x\right)^{\alpha(r-1) /(\lambda p)} \\
& \quad \leq|\rho O|^{\alpha /(\lambda p)+\alpha(r-1) /(\lambda p)}\left(\left(\frac{1}{|\rho O|} \int_{\rho O} w_{1}^{\lambda} d x\right)^{\frac{1}{\lambda r}}\left(\frac{1}{|\rho O|} \int_{\rho O}\left(\frac{1}{w_{2}}\right)^{\lambda /(r-1)} d x\right)^{\frac{r-1}{\lambda r}}\right)^{\alpha r / p} \\
& \leq C_{3}|\rho O|^{\alpha /(\lambda p)+\alpha(r-1) /(\lambda p)} \\
& \leq C_{4}|O|^{\alpha /(\lambda p)+\alpha(r-1) /(\lambda p)}
\end{aligned}
$$

It is easy to check that $(t-s) /(s t)+\alpha /(\lambda p)+\alpha(r-1) /(\lambda p)=0$, thus

$$
\begin{aligned}
\left\|G(P(\hbar))-(G(P(\hbar)))_{O}\right\|_{p, O, w_{1}^{\alpha}} & \leq C_{5}|O| \operatorname{diam}(O)|O|^{(t-s) /(s t)+\alpha /(\lambda p)+\alpha(r-1) /(\lambda p)}\|\hbar\|_{p, \rho O, w_{2}^{\alpha}} \\
& =C_{5}|O| \operatorname{diam}(O)\|\hbar\|_{p, \rho O, w_{2}^{\alpha} .}
\end{aligned}
$$

We complete the proof of Theorem 2.8. 
Definition 2.9 The weight $w(x)$ is said to satisfy the $A_{r}(\Theta)$ condition, $r>1$. Write $w \in$ $A_{r}(\Theta)$ if $w(x)>0$ a.e. and

$$
\sup _{O}\left(\frac{1}{|O|} \int_{O} w d x\right)\left(\frac{1}{|O|} \int_{O}\left(\frac{1}{w}\right)^{\frac{1}{r-1}} d x\right)^{(r-1)}<\infty
$$

for any ball $O \subset \Theta$.

If $\lambda=1, w_{1}=w_{2}$, the $A_{r, \lambda}(\Theta)$-weights reduce to the usual class of $A_{r}(\Theta)$-weights; see [3] for more details about weights. So, we have the following corollary.

Corollary 2.10 Let $\hbar \in L^{p}\left(\Theta, \wedge^{\ell}\right), \ell=0,1, \ldots, n, 1<p<\infty$, be a solution of the nonhomogeneous A-harmonic equation (1.5) in a bounded convex domain $\Theta, P$ be the potential operator defined in (1.7) with the kernel $K(x, y)$ satisfying the condition (1) of the standard estimates (2.3) and $G$ be Green's operator. Assume that $w(x) \in A_{r}(\Theta)$ for $1<r<\infty$. Then there exists a constant $C$, independent of $\hbar$, such that

$$
\left\|G(P(\hbar))-(G(P(\hbar)))_{O}\right\|_{p, O, w^{\alpha}} \leq C|O| \operatorname{diam}(O)\|\hbar\|_{p, \rho O, w^{\alpha}}
$$

for all balls $O$ with $\rho O \subset \Theta$, where $\rho>1$ and $\alpha$ are two constants with $0 \leq \alpha<1$.

\section{The $L^{\varphi}$-norm inequalities}

In this section, we first recall some definitions of elementary conceptions, including the Luxemburg norm and the class $G(p, q, C)$ of Young functions. Then we prove the $L^{\varphi}$-norm inequalities for the composite operator $G \circ P$.

A continuously increasing function $\varphi:[0, \infty) \rightarrow[0, \infty)$ with $\varphi(0)=0$ and $\varphi(\infty)=\infty$ is called an Orlicz function, and a convex Orlicz function is often called a Young function. The Orlicz space $L^{\varphi}(\Theta)$ consists of all measurable functions $f$ on $\Theta$ such that $\int_{\Theta} \varphi\left(\frac{|f|}{\chi}\right) d x<$ $\infty$ for some $\chi=\chi(f)>0$, then the nonlinear Luxemburg functional of $f$ is denoted by

$$
\|f\|_{\varphi(\Theta)}=\inf \left\{\chi>0: \int_{\Theta} \varphi\left(\frac{|f|}{\chi}\right) d x \leq 1\right\}
$$

If $\varphi$ is a Young function, then $\|\cdot\|_{\varphi(\Theta)}$ defines a norm in $L^{\varphi}(\Theta)$, which is called the Luxemburg norm.

The following class $G(p, q, C)$ is introduced in [11], which is a special class of Young functions.

Definition 3.1 We call a Young function $\varphi$ belongs to the class $G(p, q, C), 1 \leq p<q<\infty$, $C \geq 1$, if

$$
\text { (i) } \frac{1}{C} \leq \frac{\varphi\left(t^{\frac{1}{p}}\right)}{f(t)} \leq C ; \quad \text { (ii) } \quad \frac{1}{C} \leq \frac{\varphi\left(t^{\frac{1}{q}}\right)}{g(t)} \leq C
$$

for all $t>0$, where $f$ is a convex increasing function and $g$ is a concave increasing function on $[0, \infty)$. 
From [11], we assert that $\varphi, f, g$ in the above definition are doubling, namely $\varphi(2 t) \leq$ $C_{1} \varphi(t)$ for all $t>0$, and the completely similar property remains valid if $\varphi$ is replaced correspondingly with $f, g$. Besides, we have

(i) $\quad C_{2} t^{p} \leq f^{-1}(\varphi(t)) \leq C_{3} t^{p} ; \quad$ (ii) $\quad C_{2} t^{q} \leq g^{-1}(\varphi(t)) \leq C_{3} t^{q}$

where $C_{1}, C_{2}$ and $C_{3}$ are some positive constants.

$\mathrm{Bi}[1]$ constructs a special kernel function of a potential operator. Suppose the function $\phi(x)$ is defined as follows:

$$
\phi(x)= \begin{cases}\frac{1}{c} \exp \left\{\frac{1}{|x|^{2}-1}\right\}, & |x|<1, \\ 0, & |x| \geq 1\end{cases}
$$

where $c=\int_{B(0,1)} e^{\frac{1}{|x|^{2}-1}} d x$. For any $\varepsilon>0$, we write $\phi_{\varepsilon}(x)=\frac{1}{\varepsilon^{n}} \phi\left(\frac{x}{\varepsilon}\right)$. It is easy to see that $\phi(x) \in$ $C_{0}^{\infty}\left(\mathbb{R}^{n}\right)$ and

$$
\int_{\mathbb{R}^{n}} \phi_{\varepsilon}(x) d x=1
$$

Let $P$ be the potential operator in (1.7) with $K(x, y)=\phi_{\varepsilon}(x-y)$. Assume that $\hbar \in D^{\prime}\left(\Theta, \wedge^{\ell}\right)$, $\ell=0,1, \ldots, n-1$, is a differential form defined in a bounded convex domain $\Theta$ and $\hbar_{J}$ is the coefficient of $\hbar$ with supp $\hbar_{J} \subset \Theta$ for all ordered $\ell$-tuples $J$. From (4.10) in [1], we have the inequality as follows:

$$
\|d(P(\hbar))\|_{p, \Theta} \leq C\|\hbar\|_{p, \Theta},
$$

where $C$ is a constant, independent of $\hbar$, and $p$ is a positive number with $1<p<\infty$.

Now we introduce two lemmas which will be needed later.

Lemma $3.2([7])$ Let $\hbar \in D^{\prime}\left(O, \wedge^{\ell}\right), \ell=0,1, \ldots, n-1,1<p<n$, and $d \hbar \in L^{p}\left(O, \wedge^{\ell+1}\right)$. Then $\hbar-\hbar_{O}$ is in $L^{\frac{n p}{n-p}}\left(O, \wedge^{\ell}\right)$ and

$$
\left(\int_{O}\left|\hbar-\hbar_{O}\right|^{\frac{n p}{n-p}}\right)^{\frac{n-p}{n p}} \leq C_{p}(n)\left(\int_{O}|d \hbar|^{p}\right)^{1 / p}
$$

for $O$ a cube or a ball in $\mathbb{R}^{n}$.

Lemma 3.3 $([4])$ Let $\hbar \in C^{\infty}\left(\Theta, \wedge^{\ell}\right), \ell=1,2, \ldots, n-1,1<p<\infty$, and G be Green's operator. Then there exists a positive constant $C$, independent of $\hbar$, such that

$$
\begin{aligned}
& \left\|d d^{\star} G(\hbar)\right\|_{p, \Theta}+\left\|d^{\star} d G(\hbar)\right\|_{p, \Theta}+\|d G(\hbar)\|_{p, \Theta}+\left\|d^{\star} G(\hbar)\right\|_{p, \Theta}+\|G(\hbar)\|_{p, \Theta} \\
& \leq C\|\hbar\|_{p, \Theta}
\end{aligned}
$$

for any $\Theta \subset \mathbb{R}^{n}$.

Theorem 3.4 Let $\hbar \in D^{\prime}\left(\Theta, \wedge^{\ell}\right), \ell=0,1, \ldots, n-1$, be a differential form in a bounded convex domain $\Theta$ and $\hbar_{J}$ be the coefficient of $\hbar$ with supp $\hbar_{J} \subset \Theta$ for all ordered $\ell$-tuples $J$. 
Assume that $\varphi$ is a Young function in the class $G(p, q, C), 1 \leq p<q<\infty, C \geq 1$ and $q(n-p)<n p, P$ is the potential operator in (1.7) with $K(x, y)=\phi_{\varepsilon}(x-y)$ for any $\varepsilon>0$ and $G$ is Green's operator. Then there exists a constant $C$, independent of $\hbar$, such that

$$
\int_{O} \varphi\left(\left|G(P(\hbar))-(G(P(\hbar)))_{O}\right|\right) d x \leq C \int_{O} \varphi(|\hbar|) d x
$$

for all balls $O$ with $O \subset \Theta$.

Proof Using Jensen's inequality for $g^{-1}$ that is defined in Definition 3.1, (3.3), (3.2) and noticing that $\varphi$ and $g$ are doubling, for any ball $O \subset \Theta$, we obtain

$$
\begin{aligned}
& \int_{O} \varphi\left(\left|G(P(\hbar))-(G(P(\hbar)))_{O}\right|\right) d x \\
& \quad=g\left(g^{-1}\left(\int_{O} \varphi\left(\left|G(P(\hbar))-(G(P(\hbar)))_{O}\right|\right) d x\right)\right) \\
& \leq g\left(\int_{O} g^{-1}\left(\varphi\left(\left|G(P(\hbar))-(G(P(\hbar)))_{O}\right|\right)\right) d x\right) \\
& \leq g\left(C_{1} \int_{O}\left|G(P(\hbar))-(G(P(\hbar)))_{O}\right|^{q} d x\right) \\
& \leq C_{2} \varphi\left(\left(C_{1} \int_{O}\left|G(P(\hbar))-(G(P(\hbar)))_{O}\right|^{q} d x\right)^{\frac{1}{q}}\right) \\
& \leq C_{3} \varphi\left(\left(\int_{O}\left|G(P(\hbar))-(G(P(\hbar)))_{O}\right|^{q} d x\right)^{\frac{1}{q}}\right) .
\end{aligned}
$$

By Lemma 3.3, it follows that

$$
\|G(\hbar)\|_{p, O} \leq C_{4}\|\hbar\|_{p, O}
$$

If $1 \leq p<n$, by assumption, we have $q<\frac{n p}{n-p}$. Noticing that the $L^{p}$-norm of $\mid G(P(\hbar))-$ $(G(P(\hbar)))_{O} \mid$ increases with $p$, using Lemma 3.2 for $G(P(\hbar))$, (3.11) and (3.6), we have

$$
\begin{aligned}
\left(\int_{O}\left|G(P(\hbar))-(G(P(\hbar)))_{O}\right|^{q} d x\right)^{\frac{1}{q}} & \leq C_{5}\left(\int_{O}\left|G(P(\hbar))-(G(P(\hbar)))_{O}\right|^{\frac{n p}{n-p}} d x\right)^{\frac{n-p}{n p}} \\
& \leq C_{6}\left(\int_{O}|d(G(P(\hbar)))|^{p} d x\right)^{\frac{1}{p}} \\
& =C_{6}\left(\int_{O}|G(d(P(\hbar)))|^{p} d x\right)^{\frac{1}{p}} \\
& \leq C_{7}\left(\int_{O}|(d(P(\hbar)))|^{p} d x\right)^{\frac{1}{p}} \\
& \leq C_{8}\left(\int_{O}|\hbar|^{p} d x\right)^{\frac{1}{p}}
\end{aligned}
$$

since the differential operator $d$ commutes with $G$. 
When $p \geq n$, we could select a strictly increasing sequence $\left\{p_{i}\right\}$ with $1 \leq p_{i}<n \leq p$ and $p_{i} \rightarrow n$ as $i \rightarrow \infty$. Noticing that $\frac{n p_{i}}{n-p_{i}} \rightarrow \infty$ with $i \rightarrow \infty$, by keeping order of the limit, there exists $i_{0}$ with $1 \leq i_{0}<\infty$ such that $p<q<\frac{n p_{i_{0}}}{n-p_{i_{0}}}$, then

$$
\begin{aligned}
\left(\int_{O}\left|G(P(\hbar))-(G(P(\hbar)))_{O}\right|^{q} d x\right)^{\frac{1}{q}} & \leq C_{9}\left(\int_{O}\left|G(P(\hbar))-(G(P(\hbar)))_{O}\right|^{\frac{n p_{i_{0}}}{n-p_{i_{0}}}} d x\right)^{\frac{n-p_{i_{0}}}{n p_{i_{0}}}} \\
& \leq C_{10}\left(\int_{O}|\hbar|^{p_{i}} d x\right)^{\frac{1}{p_{i_{0}}}} \\
& \leq C_{11}\left(\int_{O}|\hbar|^{p} d x\right)^{\frac{1}{p}}
\end{aligned}
$$

Thus, (3.12) holds for any $p, q$ with $1 \leq p<q<\infty, q(n-p)<n p$. Since $\varphi$ is increasing, from (3.10) and (3.12), we obtain

$$
\int_{O} \varphi\left(\left|G(P(\hbar))-(G(P(\hbar)))_{O}\right|\right) d x \leq C_{3} \varphi\left(C_{12}\left(\int_{O}|\hbar|^{p} d x\right)^{\frac{1}{p}}\right) .
$$

Applying (3.14), (i) in Definition 3.1, Jensen's inequality, and noticing that $\varphi$ and $f$ are doubling, we have

$$
\begin{aligned}
\int_{O} \varphi\left(\left|G(P(\hbar))-(G(P(\hbar)))_{O}\right|\right) d x & \leq C_{3} \varphi\left(C_{12}\left(\int_{O}|\hbar|^{p} d x\right)^{\frac{1}{p}}\right) \\
& \leq C_{3} f\left(C_{13}\left(\int_{O}|\hbar|^{p} d x\right)\right) \\
& \leq C_{14} \int_{O} f\left(|\hbar|^{p}\right) d x \\
& \leq C_{15} \int_{O} \varphi(|\hbar|) d x
\end{aligned}
$$

Therefore, the proof of Theorem 3.4 has been completed.

Since each of $\varphi, f$ and $g$ in Definition 3.1 is doubling, from the proof of Theorem 3.4, we have

$$
\int_{O} \varphi\left(\frac{\left|G(P(\hbar))-(G(P(\hbar)))_{O}\right|}{\chi}\right) d x \leq C \int_{O} \varphi\left(\frac{|\hbar|}{\chi}\right) d x
$$

for all balls $O$ with $O \subset \Theta$ and any constant $\chi>0$. From the definition of the Luxemburg norm and (3.16), the following inequality with the Luxemburg norm

$$
\left\|G(P(\hbar))-(G(P(\hbar)))_{O}\right\|_{\varphi(O)} \leq C\|\hbar\|_{\varphi(O)}
$$

holds under the conditions described in Theorem 3.4.

Remark Note that in Theorem 3.4, $\varphi$ may be any Young function provided it lies in the class $G\left(p, q, C_{0}\right), 1 \leq p<q<\infty, C_{0} \geq 1$. From [11], we know that the function $\varphi(t)=t^{p} \log _{+}^{\alpha} t$ 
belongs to $G\left(p_{1}, p_{2}, C\right), 1 \leq p_{1}<p<p_{2}, t>0$ and $\alpha \in \mathbb{R}$. Here $\log _{+} t$ is a piecewise function such that $\log _{+} t=1$ for $t \leq e$; otherwise, $\log _{+} t=\log t$. Moreover, if $\alpha=0$, one verifies easily that $\varphi(t)=t^{p}$ is as well in the class $G\left(p_{1}, p_{2}, C\right), 1 \leq p_{1}<p_{2}<\infty$. Therefore, fixing the function $\varphi(t)=t^{p} \log _{+}^{\alpha} t, \alpha \in \mathbb{R}$ in Theorem 3.4, we obtain the following result.

Corollary 3.5 Let $\hbar \in D^{\prime}\left(\Theta, \wedge^{\ell}\right), \ell=0,1, \ldots, n-1$, be a differential form in a bounded convex domain $\Theta$, and $\hbar_{J}$ be the coefficient of $\hbar$ with supp $\hbar_{J} \subset \Theta$ for all ordered $\ell$-tuples $J$. Assume that $\varphi=t^{p} \log _{+}^{\alpha} t, 1 \leq p<q<\infty, C \geq 1$ and $q(n-p)<n p, P$ is the potential operator in (1.7) with $K(x, y)=\phi_{\varepsilon}(x-y)$ for any $\varepsilon>0$ and $G$ is Green's operator. Then there exists a constant $C$, independent of $\hbar$, such that

$$
\begin{aligned}
& \int_{O}\left|G(P(\hbar))-(G(P(\hbar)))_{O}\right|^{p} \log _{+}^{\alpha}\left(\left|G(P(\hbar))-(G(P(\hbar)))_{O}\right|\right) d x \\
& \quad \leq C \int_{O}|\hbar|^{p} \log _{+}^{\alpha}|\hbar| d x
\end{aligned}
$$

for all balls $O$ with $O \subset \Theta$.

\section{Global inequalities}

In this section, we first recall the definition of the $L^{\varphi}$-averaging domains. Then we extend the local $L^{\varphi}$-norm inequality for the composite operator $G \circ P$ to the global case in this kind of domains.

Definition 4.1 ([12]) Let $\varphi$ be a Young function on $[0,+\infty)$ with $\varphi(0)=0$. A proper subdomain $\Theta \subset \mathbb{R}^{n}$ is called an $L^{\varphi}$-averaging domain if $|\Theta|<\infty$, and there exists a constant $C$ such that

$$
\int_{\Theta} \varphi\left(\tau\left|\hbar-\hbar_{O_{0}}\right|\right) d x \leq C \sup _{O \subset \Theta} \int_{O} \varphi\left(\sigma\left|\hbar-\hbar_{O}\right|\right) d x
$$

for some ball $O_{0} \subset \Theta$ and all functions $\hbar$ such that $\varphi(|\hbar|) \in L_{\text {loc }}^{1}(\Theta)$, where $\tau, \sigma$ are constants with $0<\tau, \sigma \leq \infty$, and the supremum is over all balls $O$ with $O \subset \Theta$.

Theorem 4.2 Let $\hbar \in D^{\prime}\left(\Theta, \wedge^{0}\right)$ and $\Theta$ be a bounded convex $L^{\varphi}$-averaging domain with supp $\hbar \subset \Theta$. Assume that $\varphi$ is a Young function in the class $G(p, q, C), 1 \leq p<q<\infty, C \geq 1$ and $q(n-p)<n p, P$ is the potential operator in $(1.7)$ with $K(x, y)=\phi_{\varepsilon}(x-y)$ for any $\varepsilon>0$ and $G$ is Green's operator. Then there exists a constant $C$, independent of $\hbar$, such that

$$
\int_{\Theta} \varphi\left(\left|G(P(\hbar))-(G(P(\hbar)))_{O_{0}}\right|\right) d x \leq C \int_{\Theta} \varphi(|\hbar|) d x
$$

where $O_{0} \subset \Theta$ is some fixed ball.

Proof Note that $\Theta$ is an $L^{\varphi}$-averaging domain and $\varphi$ is doubling. From Definition 4.1 and (3.9), we have

$$
\begin{aligned}
\int_{\Theta} \varphi\left(\left|G(P(\hbar))-(G(P(\hbar)))_{O_{0}}\right|\right) d x & \leq C_{1} \sup _{O \subset \Theta} \int_{O} \varphi\left(\left|G(P(\hbar))-(G(P(\hbar)))_{O}\right|\right) d x \\
& \leq C_{1} \sup _{O \subset \Theta}\left(C_{2} \int_{O} \varphi(|\hbar|) d x\right)
\end{aligned}
$$




$$
\begin{aligned}
& \leq C_{1} \sup _{O \subset \Theta}\left(C_{2} \int_{\Theta} \varphi(|\hbar|) d x\right) \\
& \leq C_{3} \int_{\Theta} \varphi(|\hbar|) d x .
\end{aligned}
$$

We have completed the proof of Theorem 4.2.

Similarly, by (3.1), we conclude that

$$
\left\|G(P(\hbar))-(G(P(\hbar)))_{O_{0}}\right\|_{\varphi(\Theta)} \leq C\|\hbar\|_{\varphi(\Theta)} .
$$

From the definition of the $L^{\varphi}$-averaging domains, we see that an $L^{s}$-averaging domain [13] is a special $L^{\varphi}$-averaging domain when $\varphi(t)=t^{s}$ in Definition 4.1. Hence, we have the following result in the $L^{s}$-averaging domains.

Corollary 4.3 Let $\hbar \in D^{\prime}\left(\Theta, \wedge^{0}\right)$ and $\Theta$ be a bounded convex $L^{s}$-averaging domain with supp $\hbar \subset \Theta$. Assume that $\varphi$ is a Young function in the class $G(p, q, C), 1 \leq p<q<\infty, C \geq 1$ and $q(n-p)<n p, P$ is the potential operator in (1.7) with $K(x, y)=\phi_{\varepsilon}(x-y)$ for any $\varepsilon>0$ and $G$ is Green's operator. Then there exists a constant $C$, independent of $\hbar$, such that

$$
\int_{\Theta} \varphi\left(\left|G(P(\hbar))-(G(P(\hbar)))_{O_{0}}\right|\right) d x \leq C \int_{\Theta} \varphi(|\hbar|) d x
$$

where $O_{0} \subset \Theta$ is some fixed ball.

\section{Applications}

In this section, we give some examples of applications. By Theorem 2.5, we can obtain other norm inequalities for the composite operator $G \circ P$, such as Lipschitz and BMO norms. Now, we take the Lipschitz norm for example.

Definition $5.1([10])$ Let $\hbar \in L_{\text {loc }}^{1}\left(\Theta, \wedge^{\ell}\right), \ell=0,1, \ldots, n$. We write $\hbar \in \operatorname{locLip}_{k}\left(\Theta, \wedge^{\ell}\right), 0 \leq$ $k \leq 1$, if

$$
\|\hbar\|_{\operatorname{locLip}_{k}, \Theta}=\sup _{\rho O \subset \Theta}|O|^{-(n+k) / n}\left\|\hbar-\hbar_{O}\right\|_{1, O}<\infty
$$

for some $\rho \geq 1$. Further, we write $\operatorname{Lip}_{k}\left(\Theta, \wedge^{\ell}\right)$ for those forms whose coefficients are in the usual Lipschitz space with exponent $k$ and write $\|\hbar\|_{\operatorname{Lip}_{k}, \Theta}$ for this norm.

Theorem 5.2 Let $\hbar \in L^{p}\left(\Theta, \wedge^{\ell}\right), \ell=0,1, \ldots, n, 1<p<\infty$, be a differential form in a bounded domain $\Theta, P$ be the potential operator defined in (1.7) with the kernel $K(x, y)$ satisfying the condition (1) of the standard estimates (2.3) and G be Green's operator. Then there exists a constant $C$, independent of $\hbar$, such that

$$
\|G(P(\hbar))\|_{\operatorname{locLip}_{k}, \Theta} \leq C\|\hbar\|_{p, \Theta},
$$

where $k$ is a constant with $0 \leq k \leq 1$. 
Proof From Theorem 2.5, we have

$$
\left\|G(P(\hbar))-G(P(\hbar))_{O}\right\|_{p, O} \leq C_{1}|O| \operatorname{diam}(O)\|\hbar\|_{p, O}
$$

for all balls $O$ with $O \subset \Theta$. Using the Hölder inequality with $1=1 / p+(p-1) / p$, we get that

$$
\begin{aligned}
\left\|G(P(\hbar))-G(P(\hbar))_{O}\right\|_{1, O} & =\int_{O}\left|G(P(\hbar))-G(P(\hbar))_{O}\right| d x \\
& \leq\left(\int_{O}\left|G(P(\hbar))-G(P(\hbar))_{O}\right|^{p} d x\right)^{1 / p}\left(\int_{O} 1^{p /(p-1)} d x\right)^{(p-1) / p} \\
& =|O|^{1-1 / p}\left\|G(P(\hbar))-G(P(\hbar))_{O}\right\|_{p, O} \\
& =|O|^{1-1 / p}\left(C_{1}|O| \operatorname{diam}(O)\|\hbar\|_{p, O}\right) \\
& \leq C_{2}|O|^{2-1 / p+1 / n}\|\hbar\|_{p, O}
\end{aligned}
$$

where we have used $\operatorname{diam}(O)=C|O|^{1 / n}$. Note that the definition of Lipschitz norm, (5.4) and $2-1 / p+1 / n-1-k / n=1-1 / p+1 / n-k / n>0$ yield

$$
\begin{aligned}
\|G(P(\hbar))\|_{\operatorname{locLip}_{k}, \Theta} & =\sup _{\rho O \subset \Theta}|O|^{-(n+k) / n}\left\|G(P(\hbar))-G(P(\hbar))_{O}\right\|_{1, O} \\
& \leq \sup _{\rho O \subset \Theta}|O|^{-1-k / n} C_{2}|O|^{2-1 / p+1 / n}\|\hbar\|_{p, O} \\
& =\sup _{\rho O \subset \Theta} C_{2}|O|^{1-1 / p+1 / n-k / n}\|\hbar\|_{p, O} \\
& \leq \sup _{\rho O \subset \Theta} C_{2}|\Theta|^{1-1 / p+1 / n-k / n}\|\hbar\|_{p, O} \\
& \leq C_{3} \sup _{\rho O \subset \Theta}\|\hbar\|_{p, O} \\
& \leq C_{3}\|\hbar\|_{p, \Theta} .
\end{aligned}
$$

Thus, we have finished the proof of Theorem 5.2.

Next, we would like to use Theorem 3.4 to make some estimate.

Example 5.3 For $n \geq 2$, let $\hbar$ be a 1 -form defined in $\mathbb{R}^{n}$ by

$$
\begin{aligned}
\hbar= & \frac{x_{1}}{\sqrt{x_{1}^{2}+x_{2}^{2}+\cdots+x_{n}^{2}}} d x_{1}+\frac{x_{2}}{\sqrt{x_{1}^{2}+x_{2}^{2}+\cdots+x_{n}^{2}}} d x_{2} \\
& +\cdots+\frac{x_{n}}{\sqrt{x_{1}^{2}+x_{2}^{2}+\cdots+x_{n}^{2}}} d x_{n}
\end{aligned}
$$

and $\varphi(t)=t^{p} \log _{+} t(t>0)$, where $x=\left(x_{1}, \ldots, x_{n}\right) \in O \subset \mathbb{R}^{n} \backslash(0, \ldots, 0)$. Green's operator $G$ and the potential operator $P$ are defined as in Theorem 3.4.

It is easy to get that $|\hbar|=1$ and $\varphi$ belongs to the class $G\left(p_{1}, p_{2}, C\right), 1 \leq p_{1}<p_{2}<$ $\infty$. Although it is very difficult to compute $\int_{O}\left|G(P(\hbar))-(G(P(\hbar)))_{O}\right|^{p} \log _{+}(\mid G(P(\hbar))-$ 
$\left.(G(P(\hbar)))_{O} \mid\right) d x$ directly, we could valuate its upperbound by (3.9). Now we carry on the process as follows

$$
\begin{aligned}
\int_{O}\left|G(P(\hbar))-(G(P(\hbar)))_{O}\right|^{p} \log _{+}\left(\left|G(P(\hbar))-(G(P(\hbar)))_{O}\right|\right) d x & \leq C_{1} \int_{O}|\hbar|^{p} \log _{+}|\hbar| d x \\
& =C_{1} \int_{O} 1^{p} \log _{+} 1 d x \\
& =C_{1}|O| .
\end{aligned}
$$

Remark It is well known that uniform domains and John domains are the special $L^{\varphi}$-averaging domains. Therefore, the result of Theorem 4.2 remains valid for uniform domains and John domains.

\section{Competing interests}

The authors declare that they have no competing interests.

\section{Authors' contributions}

ZD finished the proof and the writing work. YX and YW gave ZD some excellent advice on the proof and writing. SD gave ZD lots of help in revising the paper. All authors read and approved the final manuscript.

\section{Author details}

${ }^{1}$ Department of Mathematics, Harbin Institute of Technology, Harbin, China. ${ }^{2}$ Department of Mathematics, Seattle University, Seattle, WA 98122, USA.

\section{Acknowledgements}

The authors wish to thank the anonymous referees for their time and thoughtful suggestions.

Received: 19 July 2012 Accepted: 9 November 2012 Published: 27 November 2012

\section{References}

1. $\mathrm{Bi}, \mathrm{H}$ : Weighted inequalities for potential operators on differential forms. J. Inequal. Appl. 2010, Article ID 713625 (2010)

2. Xing, Y, Ding, S: Inequalities for Green's operator with Lipschitz and BMO norms. Comput. Math. Appl. 58, 273-280 (2009)

3. Agarwal, RP, Ding, S, Nolder, CA: Inequalities for Differential Forms. Springer, New York (2009)

4. Scott, C: $L^{p}$-theory of differential forms on manifolds. Trans. Am. Math. Soc. 347, 2075-2096 (1995)

5. Ding, S: Norm estimates for the maximal operator and Green's operator. Dyn. Contin. Discrete Impuls. Syst., Ser. A Math. Anal. 16, 72-78 (2009)

6. Agarwal, RP, Ding, S: Inequalities for Green's operator applied to the minimizers. J. Inequal. Appl. 2011, 66 (2011)

7. Iwaniec, T, Lutoborski, A: Integral estimates for null Lagrangians. Arch. Ration. Mech. Anal. 125, 25-79 (1993)

8. Martell, JM: Fractional integrals, potential operators and two-weight, weak type norm inequalities on spaces of homogeneous type. J. Math. Anal. Appl. 294, 223-236 (2004)

9. Uribe, DC, Pérez, C: Two-weight, weak-type norm inequalities for fractional integrals, Calderón-Zygmund operators and commutators. Indiana Univ. Math. J. 49(2), 697-721 (2000)

10. Nolder, CA: Hardy-Littlewood theorems for A-harmonic tensors. III. J. Math. 43, 613-631 (1999)

11. Buckley, SM, Koskela, P: Orlicz-Hardy inequalities. III. J. Math. 48, 787-802 (2004)

12. Ding, $S: L^{\varphi}(\mu)$-averaging domains and the quasi-hyperbolic metric. Comput. Math. Appl. 47, 1611-1618 (2004)

13. Staples, SG: $L^{P}$-averaging domains and the Poincaré inequality. Ann. Acad. Sci. Fenn., Ser. A 1 Math. 14, 103-127 (1989)

doi:10.1186/1029-242X-2012-271

Cite this article as: Dai et al.: Inequalities for the composition of Green's operator and the potential operator. Journal of Inequalities and Applications 2012 2012:271. 